\title{
Are overexpressed alternative survivin transcripts in human bladder cancer suitable targets for siRNA-mediated in vitro inhibition?
}

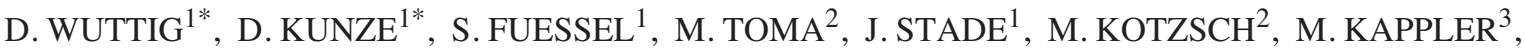 \\ H. TAUBERT ${ }^{4}$, B. SCHWENZER ${ }^{5}$, G. BARETTON ${ }^{2}$, O.W. HAKENBERG ${ }^{1}$, A. MEYE $^{1}$ and M.P. WIRTH ${ }^{1}$ \\ ${ }^{1}$ Department of Urology, ${ }^{2}$ Institute of Pathology, Technical University of Dresden, Fetscherstr. 74, D-01307 Dresden; \\ ${ }^{3}$ Department of Radiotherapy, ${ }^{4}$ Institute of Pathology, Martin-Luther-University Halle-Wittenberg, Magdeburger Str. 14, \\ D-06097 Halle (Saale); ${ }^{5}$ Institute of Biochemistry, Technical University of Dresden, Bergstr. 66, D-01069 Dresden, Germany
}

Received December 28, 2006; Accepted January 20, 2007

\begin{abstract}
In order to reduce side effects of survivin-inhibiting anticancer therapies, we determined the expression of the survivin transcripts survivin-wild-type (survivin-wt), survivin$\Delta \mathrm{Ex} 3(\Delta \mathrm{Ex} 3)$ and survivin-2B $(2 \mathrm{~B})$ in cryo-preserved tumor and non-malignant bladder tissues (18 tumor and 22 nonmalignant samples, including 17 autologous tissue pairs) by quantitative PCR. Furthermore, we investigated the biological effects following specific inhibition of the alternative transcripts $\triangle \mathrm{Ex} 3$ and $2 \mathrm{~B}$ in bladder cancer (BCa) cells. In $\mathrm{BCa}$ and non-malignant bladder tissues survivin-wt was the quantitatively dominant transcript followed by $\triangle \mathrm{Ex} 3$ and $2 \mathrm{~B}$. The mean mRNA expression of $\Delta \mathrm{Ex} 3(0.37 \mathrm{vs} .0 .06 \mathrm{zmol} / \mathrm{amol} \mathrm{GAPDH}$, respectively) and 2B (0.13 vs. $0.01 \mathrm{zmol} / \mathrm{amol} \mathrm{GAPDH}$, respectively) was significantly higher in $\mathrm{BCa}$ compared to non-malignant bladder tissues, indicating their accessibility for an expression inhibition in BCa cells. Effective and long-lasting small interfering RNA-mediated inhibition of one alternative survivin transcript caused lower cell growth reduction effects (apoptosis induction, cell cycle arrest, colony formation) compared to simultaneous inhibition of multiple survivin transcripts including survivin-wt. Inhibition of one alternative survivin transcript increased the apoptosis rate by $11 \%$ vs. $33-46 \%$ when reducing several survivin transcripts. We observed no G2/M arrest or reduction of cell colony formation after inhibiting one alternative survivin transcript. Reduction of cell viability by the chemotherapeutics cisplatin,
\end{abstract}

Correspondence to: Daniela Wuttig, Department of Urology, Faculty of Medicine, Technical University of Dresden, Fetscherstrasse 74, D-01307 Dresden, Germany

E-mail: daniela.wuttig@uniklinikum-dresden.de

\section{${ }^{*}$ Contributed equally}

Key words: small interfering RNAs, survivin- $\Delta \mathrm{Ex} 3$, survivin-2B, $\mathrm{BCa}$ mitomycin $\mathrm{C}$ or gemcitabine was stronger in combination with inhibition of several survivin transcripts than in combination with the reduction of one alternative survivin splice variant. Furthermore, reducing one alternative transcript caused chemosensitization to only one chemotherapeutic agent in contrast to inhibition of several survivin transcripts. Therefore, the alternative survivin transcripts $\Delta \mathrm{Ex} 3$ and $2 \mathrm{~B}$ do not represent reasonable targets for anticancer, at least $\mathrm{BCa}$, treatment.

\section{Introduction}

Survivin (baculoviral IAP repeat-containing protein 5, BIRC5), a potent member of the inhibitor of apoptosis (IAP) gene family, mediates several antiapoptotic functions (1-5). Survivin is also involved in cell cycle progression; as a member of the chromosomal passenger complex it is indispensable to the correct course of cytokinesis $(1,2,5-7)$. As the fourth most common transcript that is overexpressed in numerous tumor entities, survivin is one of the most attractive targets in cancer therapy (1). In many cancers, survivin expression is associated with disease progression and poor prognosis (8-11). Survivin is also involved in the development of resistance to apoptosisinducing agents $(5,8)$. In bladder cancer $(\mathrm{BCa})$, which is, with almost 61500 estimated new cases (12) and 13000 estimated tumor-related deaths in 2006 in the USA (12), one of the most common malignancies, increased survivin mRNA (13) and protein expression $(14,15)$ is associated with increased tumor grade and stage. Moreover, survivin expression serves as an independent prognostic factor of disease-free survival in patients with superficial and invasive $\mathrm{BCa}(13,15)$. Furthermore, survivin mRNA detection in urine is a specific marker for BCa $(13,16)$. Like other tumors, BCa develops resistance to conventional therapies, such as chemotherapy (17). Since overexpression of apoptosis inhibitors, such as survivin, is involved in this process, their knock-down presents a useful tool for new anticancer interventions $(17,18)$.

Although the role of survivin-wt is widely understood, little is known about the function and impact of its alternative splice variants, especially in BCa. Until now, four alternatively spliced transcripts of survivin have been described $(5,9,19,20)$. 
Two of them, $\Delta \mathrm{Ex} 3$ and $2 \mathrm{~B}$, are expressed in different tumor entities $(9,21,22) . \triangle E x 3$ mRNA lacks exon 3 and has a frameshift within exon 4 , resulting in a $15.6-\mathrm{kDa}$ protein (Fig. 1) (19). An in-frame insertion of a cryptic exon $2 \mathrm{~B}$ between exons 2 and 3 is found in $2 \mathrm{~B}$ mRNA, resulting in an 18.6-kDa protein (Fig. 1) (19,23). Different structures of the survivin protein isoforms are responsible for different functions and cellular localization patterns $(5,24)$. The function of alternative survivin splice variants is not yet completely understood. Expression of $\Delta \mathrm{Ex} 3$ decreased apoptosis rates in HepG2 hepatocellular carcinoma cells after methotrexate treatment, whereas the apoptosis rate was increased by $2 \mathrm{~B}$ expression (19). These findings suggested an antiapoptotic function for $\triangle \mathrm{Ex} 3$ and a non-antiapoptotic or even proapoptotic one for 2B. Therefore, 2B might represent a natural antagonist of anti-apoptotically acting survivin isoforms (19). Heterodimers of survivin-wt and its alternative splice variants are supposed to modulate cellular localization of the wild-type protein, such as localization of the complexes to mitochondria (24). Furthermore, co-expression of survivin-wt and $\Delta \mathrm{Ex} 3$ offered synergistic apoptosis-inhibiting effects in the Daoy medulloblastoma cell line (24). If the function of survivin-wt depends, at least in part, on these heterodimerizations, then inhibition of alternative splice variants may provide a useful therapeutic approach to apoptosis induction. Moreover, since different functions of survivin isoforms may result in different roles in tumor progression, their specific inhibition might cause antiproliferative effects.

Current survivin-inhibiting strategies, such as using small interfering RNAs (siRNAs) or antisense oligodeoxynucleotides (AS-ODN), target different survivin transcripts simultaneously (including survivin-wt). This resulted in remarkable antiproliferative effects, sensitization to apoptosis-inducing treatments as well as tumor growth reduction in vitro $(8,25,26)$ and in vivo $(8,27,28)$. siRNA- or AS-ODN-mediated survivin knock-down in different $\mathrm{BCa}$ cell lines caused increased apoptosis rates and cell cycle arrest, reduced viability and clonogenic survival as well as chemosensitization and radiosensitization (29-31). These observations supported survivin's suitability to be a therapeutic target in anti-BCa treatment.

Nevertheless, survivin expression during the G2/M phase of the cell cycle in normal cells (25) indicates its importance in normal cellular physiology. Furthermore, there is growing evidence showing involvement of survivin in hematopoietic cell maturation (32). Therefore, inhibition of survivin might result in undesirable effects on normal cells. The knock-down of one specific survivin transcript may provide a tool for more selective therapeutic approaches.

Therefore, the aim of this study was to investigate therapeutic efficiency of the specific knock-down of one alternative survivin transcript in comparison to inhibiting different survivin transcripts simultaneously by siRNAs in a suitable in vitro BCa model.

\section{Materials and methods}

Tissue samples. Tumor and non-malignant bladder tissue samples were obtained from radical cystectomy specimens of BCa patients. Twenty-four autologous, paraffin-embedded BCa tissue pairs (1 pT1,2 pT2, 16 pT3, 5 pT4; all high-grade)
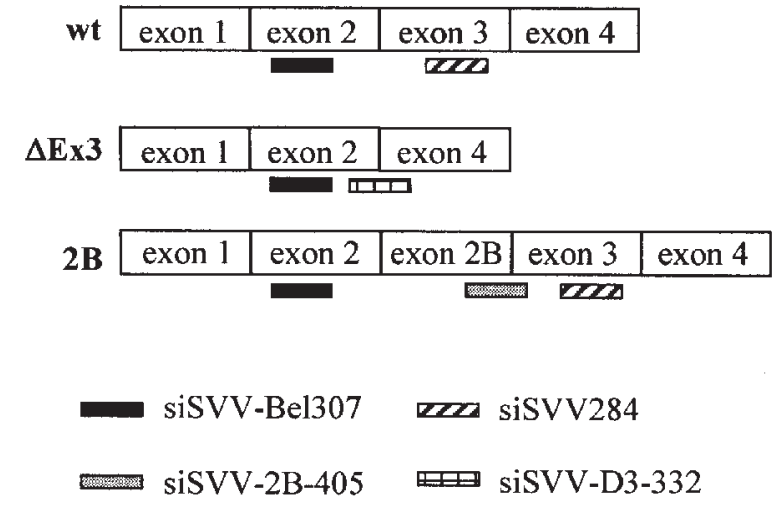

Figure 1. Scheme of the siRNA target sites within the transcripts survivinwt, $\Delta \mathrm{Ex} 3$ and $2 \mathrm{~B}$.

were used for immunohistochemical survivin staining. In addition, 18 BCa (1 pT1, 2 pT2, 10 pT3, 5 pT4; all high-grade) and 22 non-malignant cryo-preserved bladder tissue samples (thereof 17 autologous paired samples) of altogether $23 \mathrm{BCa}$ patients were used for mRNA expression analyses. Representative hemalaun and eosin (H\&E)-stained sections ( $4 \mu \mathrm{m})$ of each sample were examined by a pathologist to ensure, that the tumor cell count was $\geq 50 \%$ for malignant and $\leq 5 \%$ for non-malignant tissue samples.

Cell culture, siRNA and chemotherapeutic treatments. All cell lines were cultivated under standard conditions $\left(37^{\circ} \mathrm{C}\right.$, humidified atmosphere containing $5 \% \mathrm{CO}_{2}$ ) in DMEM (Invitrogen, Karlsruhe, Germany), supplemented with $1 \%$ non-essential amino acids, $10 \%$ fetal calf serum and $1 \%$ HEPES. The cell line EJ28 was selected as a human BCa model since it showed a remarkable expression of survivinwt and its alternative splice variants.

Three days after seeding in appropriate culture plates, cells were transfected with a mixture consisting of $200 \mathrm{nM}$ siRNA (Table I and Fig. 1) and DOTAP (ratio 1:3 w/w; Roche Molecular Biochemicals, Mannheim, Germany), each diluted in serum-free OptiMEM (Invitrogen). This transfection mixture was incubated at $37^{\circ} \mathrm{C}$ for $4 \mathrm{~h}$, cells were washed with PBS and cultivated in fresh culture medium until further analyses. Antisurvivin-siRNAs were synthesized by Eurogentec (Seraing, Belgium), non-silencing (ns)-siRNA by Qiagen (Hilden, Germany). ns-siRNA-treated cells served as a control for all examinations.

For chemosensitization experiments, $24 \mathrm{~h}$ after siRNA transfection chemotherapeutics (diluted in culture medium) were added at different concentrations, chosen in pre-tests. Mitomycin C (MMC; 0.3 or $0.9 \mu \mathrm{g} / \mathrm{ml}$ ) was incubated for $2 \mathrm{~h}$, cisplatin (CDDP; 0.7 or $2.1 \mu \mathrm{g} / \mathrm{ml}$ ) and gemcitabine (GEM; $2.5 \mathrm{ng} / \mathrm{ml}$ ) were incubated for $24 \mathrm{~h}$. Afterwards cells were washed with PBS and cultivated until viability measurements.

Expression analyses

Immunohistochemistry. Paraffin sections of autologous BCa tissue pairs $(4 \mu \mathrm{m})$ were deparaffinized and rehydrated. Endogenous peroxidase activity was blocked with hydrogen peroxide $(1.35 \%$ in methanol, $10 \mathrm{~min})$ followed by sample pulping $\left(0.01 \mathrm{M}\right.$ citrate buffer, $\left.\mathrm{pH} 6.0,15 \mathrm{~min}, 121^{\circ} \mathrm{C}\right)$. After 
Table I. siRNA constructs, their target mRNAs and sequences of their sense (s) and antisense (as) strands.

\begin{tabular}{|c|c|c|c|}
\hline siRNA construct & Survivin target-mRNA & & Target sequence $\left(5^{\prime} \rightarrow 3^{\prime}\right)$ \\
\hline \multirow[t]{2}{*}{ siSVV-Bel307 (38) } & wt (nt 307-325), 2B (nt 307-325), & $\mathrm{s}$ & GGA GCU GGA AGG CUG GGA Gdtdt \\
\hline & $\Delta \operatorname{Ex} 3($ nt $307-325)$ & as & CUC CCA GCC UUC CAG CUC Cdtdt \\
\hline \multirow[t]{2}{*}{ siSVV284 (30) } & wt (nt 358-376), 2B (nt 427-445) & $\mathrm{s}$ & GCA UUC GUC CGG UUG CGC Udtdt \\
\hline & & as & AGC GCA ACC GGA CGA AUG Cdtdt \\
\hline \multirow[t]{2}{*}{ siSVV-D3-332 } & $\Delta \mathrm{Ex} 3($ nt $332-350)$ & $\mathrm{s}$ & GAC GAC CCC AUG CAA AGG Adtdt \\
\hline & & as & UCC UUU GCA UGG GGU CGU Cdtdt \\
\hline \multirow[t]{2}{*}{ siSVV-2B-405 } & 2B (nt 405-423) & $\mathrm{s}$ & UCA CGA GAG AGG AAC AUA Adtdt \\
\hline & & as & UUA UGU UCC UCU CUC GUG Adtdt \\
\hline \multirow[t]{2}{*}{ ns-siRNA } & No complementary nucleotide & $\mathrm{s}$ & UUC UUC GAA CGU GUC ACG Udtdt \\
\hline & sequence to human mRNAs & as & ACG UGA CAC GUU CGG AGA Adtdt \\
\hline
\end{tabular}

Data in brackets represent the complementary single-strand motif within the corresponding mRNA sequence (nt, nucleotide position). mRNA sequences were taken from the NCBI database: survivin-wt nm_001168, $\Delta \mathrm{Ex} 3 \mathrm{~nm} \_001012270$ and 2B nm_001012271. siSVV-D3-332 and siSVV-2B-405 were designed by M.K. (Kappler et al, unpublished data). Because of its $100 \%$ complementary to human EPR1 (effector cell peptidase receptor 1) mRNA, application of a survivin-wt specific siRNA was not possible.

PBS washing samples were incubated for $1 \mathrm{~h}$ with a polyclonal anti-survivin-wt antibody (1:2000; NB 500-201; Novus Biologicals, Littleton, USA) and subsequently stained using Vectastain HRP Kit (Vector, Burlingame, USA) following the manufacturer's instructions. Staining reaction with $1 \mathrm{mg} / \mathrm{ml}$ diaminobenzidine (Dako, Hamburg, Germany) in PBS (+0.02\% hydrogen peroxide) was controlled microscopically followed by counterstaining of nuclei with hemalaun and dehydration. For semiquantitative analyses only nuclear survivin staining was examined. Each stained nucleus was classified as survivinpositive, regardless of staining intensity. Survivin staining was evaluated by scoring the percentage of positively stained nuclei in every tissue sample: 1 (0-19\%), 2 (20-49\%), 3 (50-69\%), $4(70-89 \%), 5(90-100 \%)$. Three visual fields per sample (magnification $\mathrm{x} 100$ ) were analyzed microscopically and then averaged. These mean values were used for calculating median and mean score of the whole sample collective.

Western blot analyses. Cells (25000 cells per sample) were lysed and proteins were separated on a $12 \%$ SDS polyacrylamide gel and blotted onto a nitrocellulose membrane. Immunostaining was performed according to standard protocols, using bovine serum albumin (BSA) diluted in PBS with $0.1 \%$ Tween-20 for blocking membrane (5\% BSA), dissolving antibodies $(0.5 \%$ BSA) and washing steps $(0.5 \%$ BSA). The following antibodies were used: anti-survivin-wt (1:1000; NB 500-201; Novus Biologicals), anti-2B (1:500; ab3729; Abcam, Cambridge, UK), anti- $\Delta \mathrm{Ex} 3$ (1:1000; ab3731; Abcam), HRP-linked anti-mouse immunoglobulin (1:1000; P 0260; Dako) and HRP-linked anti-rabbit immunoglobulin (1:1000; P 0217; Dako). Detection was carried out using the Enhanced Chemiluminescence (ECL) Kit (Amersham, Freiburg, Germany). As a control for equal loading, B-actin was detected by a monoclonal antibody (1:10000; A 5316; Sigma, St. Louis, MO, USA). Protein levels were quantified by densitometry using Intas Gelscan software (Goettingen, Germany) and survivin protein levels were normalized to those of $\beta$-actin.

Quantitative real-time PCR. RNA from cells and tissue samples was isolated using standard protocols (Invisorb ${ }^{\circledR}$ Spin Cell/Tissue RNA Mini Kit; Invitek, Berlin, Germany) and reversely transcribed into cDNA (SuperScript ${ }^{\mathrm{TM}}$ II RNase H-Reverse Transcriptase Kit; Invitrogen). Transcript levels of the different survivin forms and glyceraldehyde-3-phosphate dehydrogenase (GAPDH) were quantified by quantitative real-time PCR (Roboscreen, Leipzig, Germany). Survivin expression values were calculated in zmol survivin per amol GAPDH. Tissue samples were excluded from analyses of a transcript, if the mean deviation within a dependently repeated quantification of that transcript exceeded $35 \%$.

\section{Biological effects of siRNA treatment}

Viability. Cellular viability was examined using cell proliferation reagent WST-1 (Roche Molecular Biochemicals) following the manufacturer's instructions. For further analyses, cells were washed with PBS and cultivated in fresh medium. Viabilities were determined 72 and $96 \mathrm{~h}$ after siRNA transfection. Viabilities of siRNA mono-treatments and siRNA/ chemotherapeutic combination treatments were normalized to those of ns-siRNA. Viabilities following chemotherapeutic mono-treatments were normalized to those of untreated control.

Colony formation. Cells were harvested $48 \mathrm{~h}$ after siRNA transfection, 100 cells were seeded in 6-well plates ( 3 wells per sample), cultivated and Giemsa stained after 10-12 days of cultivation. Macroscopically visible colonies were counted. Colony formation for each sample was calculated as the mean value of the three examined wells. 

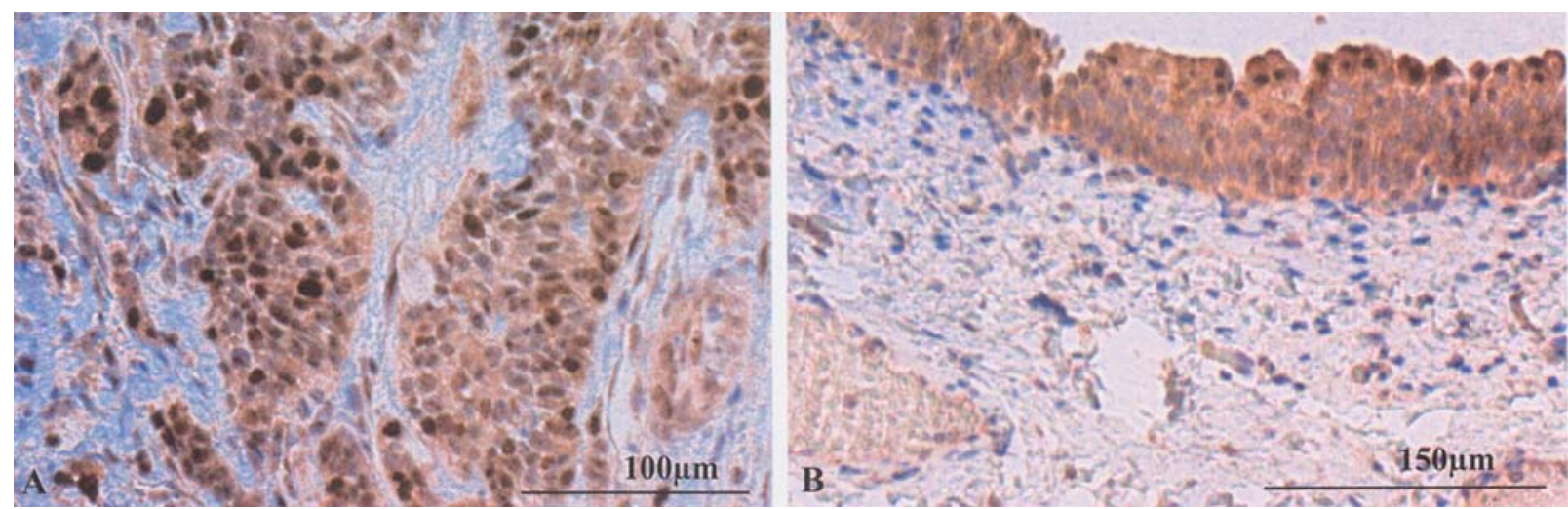

Figure 2. Representative immunohistochemical staining of survivin-wt. A, BCa tissue (T4, high-grade). B, non-malignant bladder tissue (high survivin expression only in urothelium); blue, nuclei; brown, survivin-wt protein.
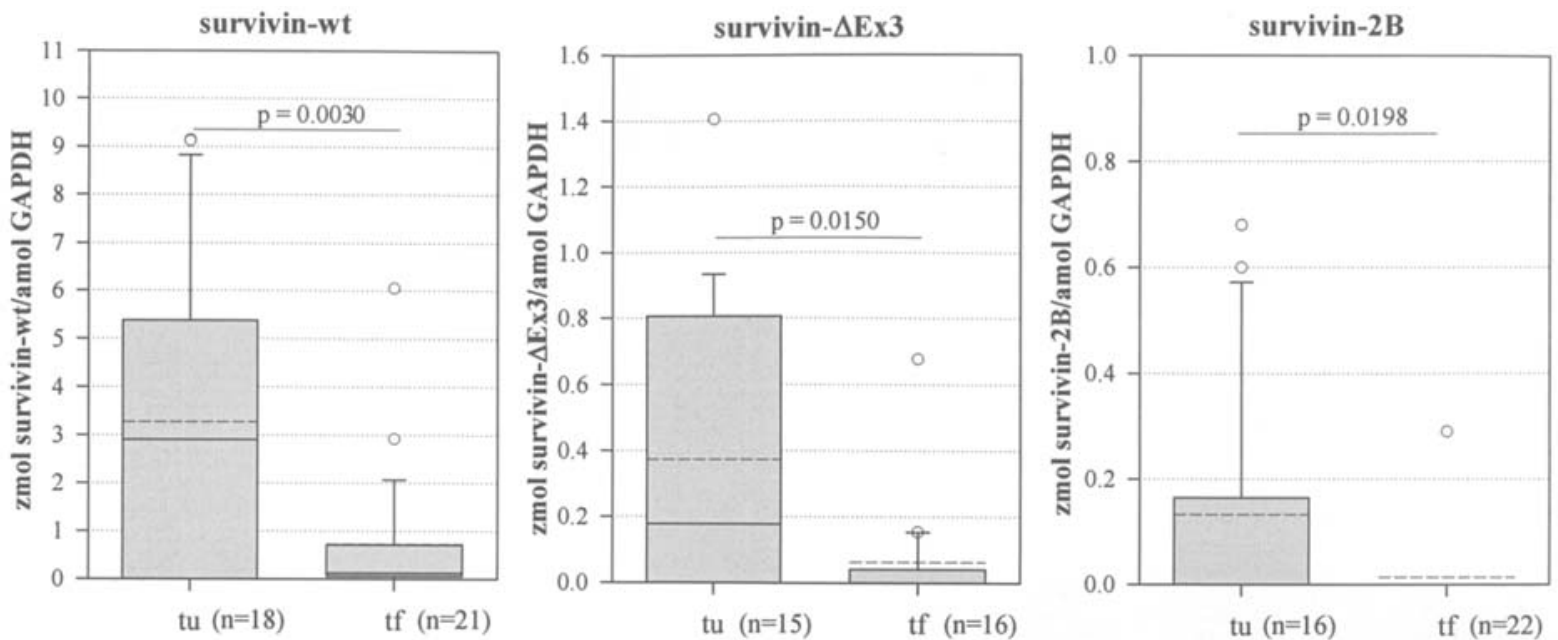

Figure 3. mRNA expression of survivin-wt, $\triangle \mathrm{Ex} 3$ and $2 \mathrm{~B}$ in $\mathrm{BCa}(\mathrm{tu})$ and non-malignant tissue samples (tf) of radical cystectomy specimens. Box plots represent values from 25 to $75 \%$, error bars represent values from 10 to $90 \%$, respectively. Dashed lines within the box plots show the mean values and solid lines show the median values. Points outside the error bars display outliers. Because of excluding values with a mean deviation $>35 \%$, different numbers of samples ( $\mathrm{n}$ ) were used for calculation. Expression of all transcripts was significantly higher in tumor tissues than in non-malignant tissues (p<0.05). Statistical significance was examined by Student's t-test.

Apoptosis and cell cycle. Apoptosis rates were assessed using Annexin V-FITC Apoptosis Detection Kit and cell cycle analyses were performed using Cycle Test Plus DNA Reagent Kit (both BD Biosciences, Heidelberg, Germany) according to the manufacturer's instructions.

Statistical analyses. Statistical analyses were performed using an unpaired two-sided (homoscedastic) Student's t-test. Statistical significance was determined at $\mathrm{p}<0.05$.

\section{Results}

Survivin expression in human bladder tissue samples

Protein expression. In immunohistochemical analyses both cytoplasmatic and nuclear survivin-wt was detected. Cells of tumor samples showed a staining of the whole nucleus, whereas in non-malignant cells a punctured nuclear staining pattern was noted. All 24 examined BCa samples showed a higher number of stained nuclei and a higher staining intensity compared to autologous non-malignant bladder tissues (Fig. 2).
For $\mathrm{BCa}$ tissue staining we determined a mean score of 4.8 and a median of 5.0. For corresponding non-malignant bladder tissues the mean score was 2.8 and the median was 2.7. No association was observed between the percentage of nuclear survivin staining and tumor stage.

mRNA expression. Proportional distribution of survivin transcripts ranged from $74-100 \%$ survivin-wt, $0-16 \% \Delta \mathrm{Ex} 3$ and $0-10 \%$ 2B in $\mathrm{BCa}$ tissues. In non-malignant bladder tissue the samples' proportional distribution was $82-100 \%$ survivinwt, $0-18 \% \Delta \mathrm{Ex} 3$ and $0-4 \% 2 \mathrm{~B}$. In all examined tumor and non-malignant tissue samples, we observed the following expression patterns: survivin-w $>\Delta \mathrm{Ex} 3>2 \mathrm{~B}$ (when transcripts were detected). In tumor samples survivin-wt was detected in $12 / 17$ cases $(71 \%)$ in a range of 0.17 to $9.14 \mathrm{zmol} / \mathrm{amol}$ GAPDH (Fig. 3). Non-malignant bladder tissue samples showed a survivin-wt expression in $16 / 21$ cases $(76 \%)$ in a range of 0.07 to $6.05 \mathrm{zmol} / \mathrm{amol} \mathrm{GAPDH}$ (Fig. 3). $\Delta \mathrm{Ex} 3$ was detected in $8 / 15$ (53\%; 0.18-1.41 zmol/amol GAPDH) BCa samples and 5/17 (29\%; 0.04-0.68 zmol/amol GAPDH) non- 


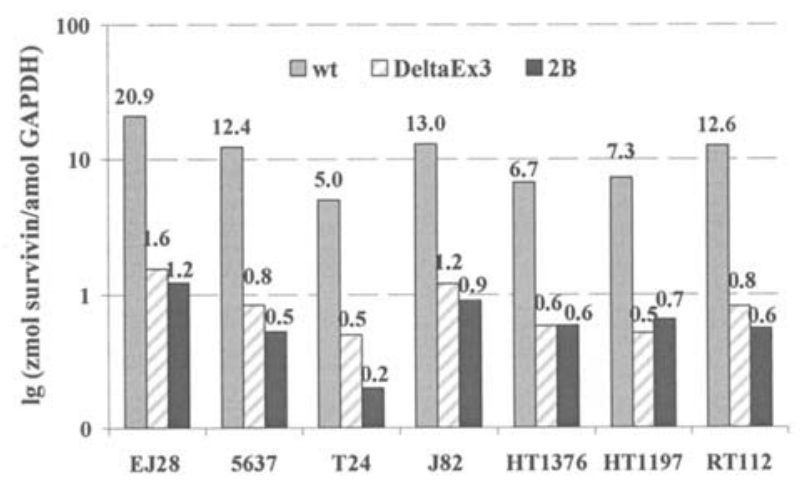

Figure 4. mRNA expression of survivin-wt and its alternative splice variants $\triangle \mathrm{Ex} 3$ and $2 \mathrm{~B}$ in human $\mathrm{BCa}$ cell lines. Digits on bars represent the precise expression values.

malignant samples (Fig. 3). We detected 2B in 7/16 (44\%; 0.07$0.68 \mathrm{zmol} / \mathrm{amol} \mathrm{GAPDH})$ tumor samples and $1 / 22(5 \% ; 0.29$ zmol/amol GAPDH) non-malignant bladder tissues (Fig. 3). The mean expression was significantly higher in malignant than in non-malignant bladder tissues for all quantified survivin transcripts (Fig. 3). Mean expression of survivin-wt was $3.27 \mathrm{zmol} /$ amol GAPDH in BCa and $0.73 \mathrm{zmol} / \mathrm{amol}$ GAPDH in nonmalignant bladder tissues. $\triangle \mathrm{Ex} 3$ showed a mean expression of $0.37 \mathrm{zmol} / \mathrm{amol}$ GAPDH in tumor and of $0.06 \mathrm{zmol} / \mathrm{amol}$ GAPDH in non-malignant samples. $2 \mathrm{~B}$ was detected with a mean expression of $0.13 \mathrm{zmol} / \mathrm{amol} \mathrm{GAPDH}$ in $\mathrm{BCa}$ and $0.01 \mathrm{zmol} / \mathrm{amol}$ GAPDH in non-malignant tissues. Within autologous tissue samples survivin-wt was overexpressed in tumor in $10 / 17$ cases $(59 \%), \Delta \mathrm{Ex} 3$ in $6 / 12$ cases $(50 \%)$ and $2 \mathrm{~B}$ in $7 / 15$ cases $(47 \%)$.

In vitro inhibition of survivin transcripts and its biological effects

Survivin mRNA expression in BCa cell lines. All tested BCa cell lines expressed survivin-wt and its alternative splice variants $\triangle \mathrm{Ex} 3$ and $2 \mathrm{~B}$ (Fig. 4). Proportional distribution of these transcripts in the cell lines was similar to that in human BCa tissue samples. Since EJ28 cells showed the highest expression of all examined survivin transcripts, this cell line was selected as a model system of human $\mathrm{BCa}$. The initial survivin expression in EJ28 cells amounts to 24 zmol survivin/ amol GAPDH. Survivin-wt represents $88 \%, \Delta \mathrm{Ex} 37 \%$ and 2B $5 \%$ of this total survivin.

$m R N A$ and protein expression of survivin isoforms in siRNAtreated EJ28 cells. All siRNA constructs effectively knockeddown their target transcripts 24 and $48 \mathrm{~h}$ after the start of transfection (Table II). Survivin-wt inhibition by siSVVBe1307 and siSVV284 was also observed at the protein level by Western blot analyses (Table II). siSVV-Bel307, siSVV284 and siSVV-2B-405 selectively reduced their target mRNAs, whereas siSVV-D3-332 also inhibited nontargeted survivin-wt $24 \mathrm{~h}$ after transfection started (Fig. 1 and Table II).

Biological effects of survivin inhibition in EJ28 cells. All siRNAs increased apoptosis by $11-46 \%$ (Table II). siSVVBel307 and siSVV284 increased G2/M arrest by 28 and 92\%, respectively, as well as decreased cell colony formation to 70 and $68 \%$, respectively (Table II). All these results were confirmed with an independent experimental series (data not shown).

siRNA/chemotherapeutics combination treatments. Combination treatments of siRNAs and different concentrations of CDDP, MMC or GEM showed statistically significant chemosensitization effects mainly by using siSVV-Bel307 and siSVV284 (Fig. 5). The strongest viability reduction to $52 \%$ of that of ns-siRNA/chemotherapeutic therapy was observed for siSVV284/CDDP $(2.1 \mu \mathrm{g} / \mathrm{ml})$ treatment. In contrast, the strongest effect caused by the specific inhibition of an alternative survivin transcript was a viability reduction to $83 \%$ of that of the corresponding control (siSVV-2B-405/GEM treatment). Furthermore, siSVV-Bel307 and siSVV284 significantly sensitized EJ28 cells to different chemotherapeutics, in contrast to siSVV-D3-332 and siSVV-2B-405.

\section{Discussion}

Because of its essential functions in inhibiting apoptosis and regulating cell cycle as well as its differential expression in cancer and normal cells, survivin is one of the most prominent cancer-related genes (1). Despite the intensive knowledge regarding survivin-wt, the role of its alternative splice variants

Table II. siRNA-mediated knock-down of survivin transcripts survivin-wt, $\Delta \mathrm{Ex} 3$ and $2 \mathrm{~B}$ in EJ28 cells and their effects on cell proliferation.

\begin{tabular}{|c|c|c|c|c|c|c|c|c|c|c|c|c|}
\hline \multirow{2}{*}{$\begin{array}{l}\text { siRNA } \\
\text { construct }\end{array}$} & \multicolumn{3}{|c|}{ mRNA expression ${ }^{a}$} & \multicolumn{3}{|c|}{ mRNA expression } & \multicolumn{3}{|c|}{ Protein expression } & \multirow[b]{2}{*}{ Apoptosis } & \multirow{2}{*}{$\begin{array}{l}\mathrm{G} 2 / \mathrm{M} \\
\text { arrest }\end{array}$} & \multirow{2}{*}{$\begin{array}{l}\text { Colony } \\
\text { formation }\end{array}$} \\
\hline & wt & $\Delta \mathrm{Ex} 3$ & $2 \mathrm{~B}$ & wt & $\Delta \mathrm{Ex} 3$ & $2 \mathrm{~B}$ & wt & $\Delta \mathrm{Ex} 3$ & $2 \mathrm{~B}$ & & & \\
\hline siSVV-Bel307 & -62 & -53 & -59 & -58 & -52 & -60 & -55 & \pm 0 & +17 & +46 & +28 & -30 \\
\hline siSVV284 & -79 & +38 & -70 & -78 & -5 & -81 & -86 & +1 & +77 & +33 & +92 & -32 \\
\hline siSVV-D3-332 & -16 & -26 & +43 & +10 & -10 & +44 & +74 & +10 & +118 & +11 & -7 & +1 \\
\hline siSVV-2B-405 & +23 & +73 & -100 & +68 & +96 & -72 & +50 & -8 & +11 & +11 & -3 & -14 \\
\hline
\end{tabular}

All parameters were measured $48 \mathrm{~h}\left({ }^{\mathrm{a}} 24 \mathrm{~h}\right)$ after the start of transfection. Data are normalized to those of ns-siRNA-treated cells and declared in $\%$ as differences to ns-siRNA-treated control. 


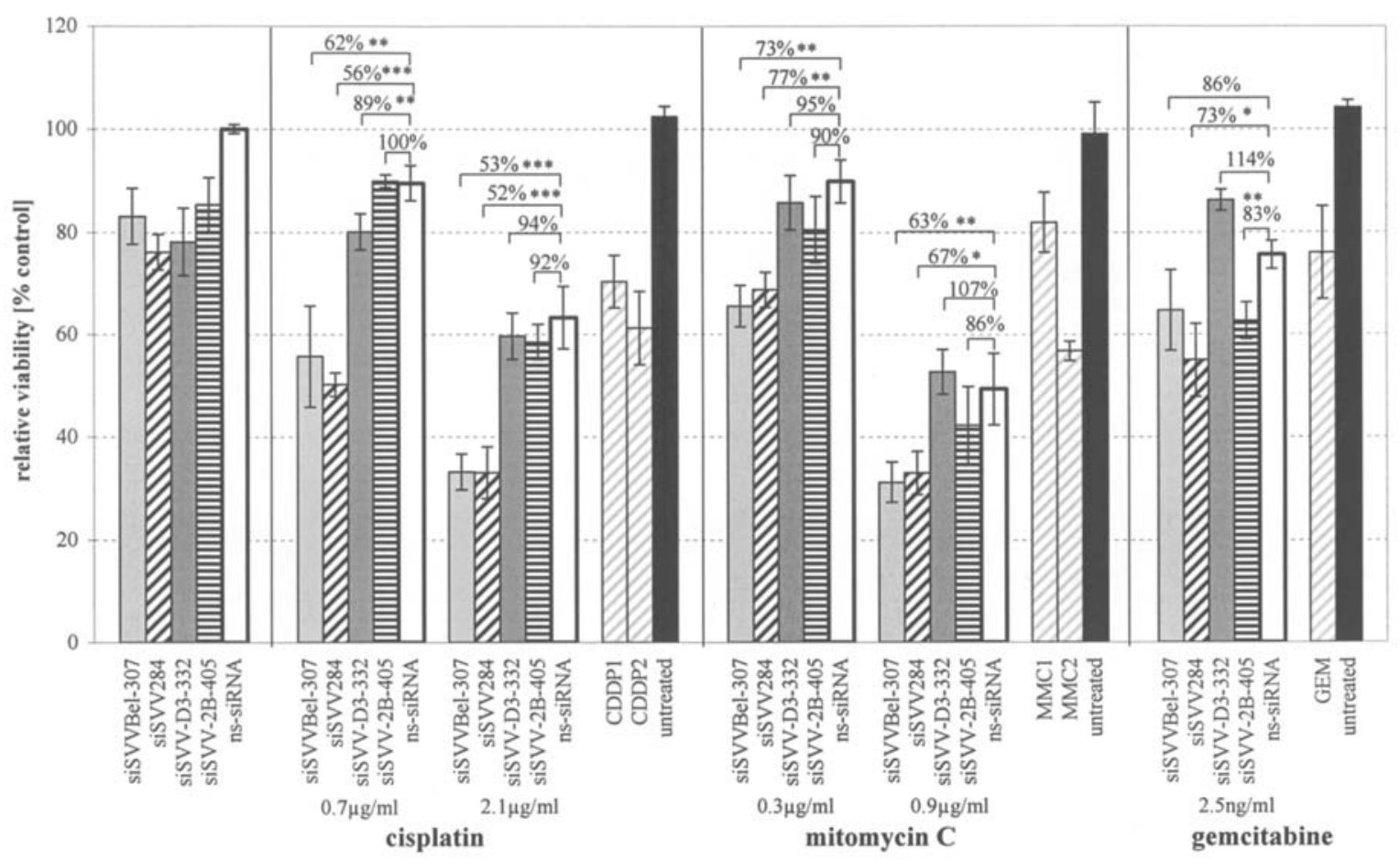

Figure 5. Viability of EJ28 cells after siRNA mono-treatments and siRNA/chemotherapeutic combination treatments. Cell viabilities following siRNA monotreatments and siRNA/chemotherapeutic combination treatments are normalized to those of ns-siRNA. Viabilities following chemotherapeutic mono-treatments are normalized to untreated control. The figure shows the strongest effect of each treatment, reproducibly observed in three independent experiment series. Statistical significance was examined by Student's t-test: ${ }^{*} \mathrm{p}<0.05 ;{ }^{* *} \mathrm{p}<0.01 ;{ }^{* * *} \mathrm{p}<0.001$

in tumor events and cancer therapy is widely unknown. In order to reduce side effects on normal cells, it appears reasonable to inhibit only one specific survivin splice variant instead of multiple ones. In the present study we investigated, if this approach could provide a suitable therapeutic strategy.

Our investigations showed an overexpression of survivinwt at both the mRNA and protein levels in malignant compared to non-malignant tissue samples obtained from radical cystectomy specimens of BCa patients as found in previous analyses $(1,13,14)$. So far, expression of alternative survivin transcripts $\triangle \mathrm{Ex} 3$ and $2 \mathrm{~B}$ has been characterized in a few tumor entities, including breast cancer $(22,33,34)$, renal cell carcinoma (35), medulloblastoma (36), soft tissue sarcomas (37) and colorectal carcinoma (21). We therefore focussed on these two alternative splice variants, whereas the other known survivin variants survivin-3B and survivin- $2 \alpha$ were not analyzed $(5,9)$. As described for the mentioned tumor entities, we found an expression of $\triangle \mathrm{Ex} 3$ and $2 \mathrm{~B}$ mRNA in $\mathrm{BCa}$ as well as in non-malignant bladder tissue samples. Survivin-wt was the dominant survivin transcript expressed in the examined tissue samples (in tumor $74-100 \%$ of total survivin), whereas $\Delta \mathrm{Ex} 3$ and 2B were detected at significantly lower levels (Fig. 3). Mean expression of all investigated survivin transcripts was significantly higher in tumors than in non-malignant bladder tissues (Fig. 3). Therefore, besides survivin-wt also its alternative transcripts $\triangle \mathrm{Ex} 3$ and $2 \mathrm{~B}$ should be accessible for an siRNA-mediated expression inhibition in BCa. Higher expression of $2 \mathrm{~B}$ in tumor samples compared to non-malignant bladder tissues did not necessarily show an association with tumor progression. Ryan et al (33) also investigated an overexpression of $2 \mathrm{~B}$ in breast cancer compared to normal breast tissue, but found no relation between $2 \mathrm{~B}$ expression and apoptosis. The detection of survivin-wt, $\Delta \mathrm{Ex} 3$ and $2 \mathrm{~B}$ mRNAs in non-malignant bladder tissues can be explained by survivin expression in the G2/M phase of strongly proliferating nonmalignant cells in the urothelium. Since most of the included BCa samples were histologically classified as pT3/pT4 G3, we are not able to report an association of survivin splice variant expression with stage or grade of BCa.

Before performing survivin inhibition experiments, we first looked for a suitable in vitro model of $\mathrm{BCa}$ by investigating expression of survivin-wt, $\triangle \mathrm{Ex} 3$ and $2 \mathrm{~B}$ in seven human $\mathrm{BCa}$ cell lines. All examined cell lines expressed these transcripts. Of these, survivin-wt was the quantitatively dominant transcript (85-90\% dependent on cell line), whereas $\Delta \mathrm{Ex} 3(5.8-8.8 \%)$ and 2B (3.5-7.7\%) were expressed at lower levels. Since EJ28 cells showed the highest expression of all examined transcripts and a similar proportional transcript distribution as BCa tissues, they were used as a model system for in vitro survivin knockdown.

For transfection experiments we used siRNAs targeting different survivin transcripts simultaneously (siSVV-Bel307, siSVV284) and also siRNAs specific for one alternative survivin splice variant (siSVV-D3-332, siSVV-2B-405) and investigated the biological effects of these treatments in two independent experiment series. We observed a selective, effective and long-lasting mRNA reduction to $38 \%$ by siSVVBel307 and to $19 \%$ by siSVV284, resulting in remarkable antiproliferative effects (Table II and Fig. 5). These findings and the magnitude of the observed effects confirm previously published data (30,38-41). Belyanskaya et al (38) reported a survivin mRNA and protein reduction by 70 and $50 \%$, 
respectively, as well as sensitization to CDDP in the SW2 small-cell lung cancer cell line. Effective inhibition of survivin mRNA and protein expression by siSVV284 resulting in antiproliferative effects was achieved in cell lines originating from several tumor entities, including colon and breast cancer (39-41). Our group previously described a siSVV284-mediated inhibition of colony formation by $60 \%$, an increase in apoptosis rate (by 30\%) and $\mathrm{G} 2 / \mathrm{M}$ arrest (by 100\%) as well as formation of multinucleated cells in different BCa cell lines, including EJ28 (30).

In the present study, we investigated for the first time the effects on cell proliferation caused by specific inhibition of the alternative survivin transcripts $\triangle \mathrm{Ex} 3$ (siSVV-D3-332) and 2B (siSVV-2B-405). Similar to siSVV-Bel307 and siSVV284, we showed a selective reduction of the target mRNA $2 \mathrm{~B}$ by siSVV-2B-405. siSVV-D3-332 offered an unspecific action by inhibition of survivin-wt (down to $84 \%$ ) in addition to that of $\Delta \mathrm{Ex} 324 \mathrm{~h}$ after the start of transfection (Table II). This construct hybridizes to 10 bases within exon 2 and 11 bases within exon 4 , all of which are also present in survivin-wt. This might have been sufficient for inhibiting survivin-wt.

Despite a selective, effective and long-lasting reduction of 2B mRNA [down-regulation of $2 \mathrm{~B}$ to $0 \% 24 \mathrm{~h}$ after siRNA treatment (Table II) was probably caused by an effective knock-down of this transcript below the detection limit], siSVV-2B-405 treatment resulted only in low antiproliferative effects in EJ28 cells. These observations support the theory of reduced antiapoptotic potential of $2 \mathrm{~B}$ in $\mathrm{BCa}$ cells, as it was described for HepG2 hepatocellular carcinoma cells (19). Proapoptotic action of 2B was also confirmed by its decreased expression in higher tumor grades of colorectal carcinoma (21) and renal cell carcinoma (35), but contradicted by a correlation of $2 \mathrm{~B}$ expression with poorer prognosis in adult acute myeloid leukaemia (42) as well as by the absence of a relation between $2 \mathrm{~B}$ expression and apoptosis in breast carcinoma (33). Therefore, the role of survivin splice variants in apoptosis and tumorigenesis or tumor progression has to be further investigated and might even depend on tumor entity or cell line.

siSVV-D3-332 caused lower mRNA inhibition effects compared to the other used constructs (Table II). This finding might explain lower biological effects following treatment with this construct. Formation of secondary structures in the target mRNA could lead to hindrance in siRNA hybridization and therefore, could be responsible for limited mRNA reduction. Specific inhibition of $\Delta \mathrm{Ex} 3$ is possible only at the transition site between exons 2 and 4 (Fig. 1). Therefore, no other siRNA constructs can be designed performing $\triangle \mathrm{Ex} 3$ specific survivin knock-down.

A pre-requisite for an effective target knock-down is not only mRNA reduction, but also reduction of the corresponding protein isoform. We observed a protein reduction only for survivin-wt; an inhibition was not demonstrated for $\Delta \mathrm{Ex} 3$ or 2B. Explanations include the possibility that lifetimes of $\triangle \mathrm{Ex} 3$ protein and of $2 \mathrm{~B}$ protein were not yet expired at detection time ( $48 \mathrm{~h}$ after transfection). It is also supposable, that inhibition of alternative splice variant mRNAs does not remarkably change protein levels of survivin isoforms. Furthermore, when only one alternative survivin transcript was knocked-down, other transcripts achieved higher expression compared to control (ns-siRNA treated) cells. This might be caused by a mechanism regulating expression of the different survivin transcripts together as described for other alternatively spliced transcripts (43). Absence of protein reduction or mRNA up-regulation could be responsible for the low effects observed following siSVV-D3-332 and siSVV-2B-405 treatments.

However, mRNA inhibition of one specific alternative survivin splice variant $(\triangle \mathrm{Ex} 3$ or $2 \mathrm{~B})$ did not result in remarkable antiproliferative effects. These treatments offered less effects in apoptosis induction, cell cycle arrest, reduction of long-term cell proliferation and chemosensitization than treatments with siSVV-Bel307 or siSVV284. These findings were confirmed by the observation that siSVV-2B-405 and siSVV-D3-332 did not radiosensitize human sarcoma cells as effectively as siSVV284 (Kappler et al, unpublished data).

In conclusion, either a simultaneous inhibition of different survivin transcripts including survivin-wt or a reduction of survivin-wt alone was necessary for strong antiproliferative effects caused by siSVV-Bel307 and siSVV284. Since survivin-wt expression is associated with the course of disease, the latter is more plausible. We have shown that the specific inhibition of one alternative survivin transcript is not reasonable for anticancer treatment, at least in $\mathrm{BCa}$ therapy.

\section{Acknowledgements}

The authors would like to express their gratitude to Katja Robel, Andrea Lohse, Jana Herrmann and Antje Zobjack for their excellent technical assistance, and to Dr Woei-Yun Siow for critically reviewing the manuscript. This study was kindly supported by a grant of the Dr Robert Pfleger-Stiftung (to S.F. and A.M.). M.K. was supported by a grant of SaxonyAnhalt (FKZ: 3584/1104M).

\section{References}

1. Altieri DC: Validating survivin as a cancer therapeutic target. Nat Rev Cancer 3: 46-54, 2003.

2. Reed JC: The survivin saga goes in vivo. J Clin Invest 108: 965-969, 2001.

3. Dohi T, Okada K, Xia F, et al: An IAP-IAP complex inhibits apoptosis. J Biol Chem 279: 34087-34090, 2004.

4. Dohi T, Beltrami E, Wall NR, Plescia J and Altieri DC: Mitochondrial survivin inhibits apoptosis and promotes tumorigenesis. J Clin Invest 114: 1117-1127, 2004.

5. Li F and Ling X: Survivin study: an update of 'What is the next wave?'. J Cell Physiol 208: 476-486, 2006.

6. Vader G, Kauw JJ, Medema RH and Lens SM: Survivin mediates targeting of the chromosomal passenger complex to the centromere and midbody. EMBO Rep 7: 85-92, 2006.

7. Carvalho A, Carmena M, Sambade C, Earnshaw WC and Wheatley SP: Survivin is required for stable checkpoint activation in taxol-treated HeLa cells. L Cell Sci 116: 2987-2998, 2003.

8. Zaffaroni N, Pennati M and Daidone MG: Survivin as a target for new anticancer interventions. J Cell Mol Med 9: 360-372, 2005.

9. Li F: Role of survivin and its splice variants in tumorigenesis. Br J Cancer 92: 212-216, 2005.

10. Monzo M, Rosell R, Felip E, et al: A novel anti-apoptosis gene: re-expression of survivin messenger RNA as a prognosis marker in non-small-cell lung cancers. J Clin Oncol 17: 2100-2104, 1999.

11. Kappler M, Koehler T, Kampf C, et al: Increased survivin transcript levels: an independent negative predictor of survival in soft tissue sarcoma patients. Int J Cancer 95: 360-363, 2001.

12. Jemal A, Siegel R, Ward E, et al: Cancer statistics, 2006. CA Cancer J Clin 56: 106-130, 2006. 
13. Weikert S, Christoph F, Schrader M, Krause H, Miller K and Mueller M: Quantitative analysis of survivin mRNA expression in urine and tumor tissue of bladder cancer patients and its potential relevance for disease detection and prognosis. Int $\mathbf{J}$ Cancer 116: 100-104, 2005.

14. Wu Y, Wang G, Wei J and Wen X: Survivin protein expression positively correlated with proliferative activity of cancer cell in bladder cancer. Indian J Med Sci 59: 235-242, 2005.

15. Ku JH, Kwak C, Lee HS, Park HK, Lee E and Lee SE: Expression of survivin, a novel inhibitor of apoptosis, in superficial transitional cell carcinoma of the bladder. J Urol 171: 631-635, 2004.

16. Moussa O, Abol-Enein H, Bissada NK, Keane T, Ghoneim MA and Watson DK: Evaluation of survivin reverse transcriptasepolymerase chain reaction for non-invasive detection of bladder cancer. J Urol 175: 2312-2316, 2006.

17. Duggan BJ, Gray S, Johnston SR, Williamson K, Miyake H and Gleave M: The role of antisense oligonucleotides in the treatment of bladder cancer. Urol Res 30: 137-147, 2002.

18. Fojo $\mathrm{T}$ and Bates $\mathrm{S}$ : Strategies for reversing drug resistance. Oncogene 22: 7512-7523, 2003.

19. Mahotka C, Wenzel M, Springer E, Gabbert HE and Gerharz CD: Survivin- $\Delta \mathrm{Ex} 3$ and survivin-2B. Two novel splice variants of the apoptosis inhibitor survivin with different antiapoptotic properties. Cancer Res 59: 6097-6102, 1999.

20. Caldas H, Honsey LE and Altura RA: Survivin $2 \alpha$ : a novel survivin splice variant expressed in human malignancies. Mol Cancer 4: 11-20, 2005.

21. Suga K, Yamamoto T, Yamada Y, Miyatake S, Nakagawa T and Tanigawa $\mathrm{N}$ : Correlation between transcriptional expression of survivin isoforms and clinicopathological findings in human colorectal carcinomas. Oncol Rep 13: 891-897, 2005.

22. Vegran F, Boidot R, Oudin C, Riedinger JM and Lizard-Nacol S: Distinct expression of survivin splice variants in breast carcinomas. Int J Oncol 27: 1151-1157, 2005.

23. Caldas H, Jiang Y, Holloway MP, Fangusaro J, Mahotka C, Conway EM and Altura RA: Survivin splice variants regulate the balance between proliferation and cell death. Oncogene 24: 1994-2007, 2005

24. Mahotka C, Liebmann J, Wenzel M, Suschek CV, Schmitt M, Gabbert HE and Gerharz CD: Differential subcellular localization of functionally divergent survivin splice variants. Cell Death Differ 9: 1334-1342, 2002.

25. Chen J, Wu W, Tahir SK, et al: Down-regulation of survivin by antisense oligonucleotides increases apoptosis, inhibits cytokinesis and anchorage-independent growth. Neoplasia 2: 235-241, 2000

26. Xia C, Xu Z, Yuan X, et al: Induction of apoptosis in mesothelioma cells by antisurvivin oligonucleotides. Mol Cancer Ther 1: 687-694, 2002.

27. Lu X, Zheng Q and Xiong J: Effect of siRNA targeting survivin gene on the biological behavior of hepatocellular carcinoma. J Huazhong Univ Sci Technolog Med Sci 25: 48-50, 2005.

28. Cao C, Mu Y, Hallahan DE and Lu B: XIAP and survivin as therapeutic targets for radiation sensitization in preclinical models of lung cancer. Oncogene 23: 7047-7052, 2004.
29. Fuessel S, Kueppers B, Ning S, et al: Systematic in vitro evaluation of survivin directed antisense oligodeoxynucleotides in bladder cancer cells. J Urol 171: 2471-2476, 2004.

30. Ning S, Fuessel S, Kotzsch M, et al: siRNA-mediated downregulation of survivin inhibits bladder cancer cell growth. Int J Oncol 25: 1065-1071, 2004.

31. Fuessel S, Herrmann J, Ning S, et al: Chemosensitization of bladder cancer cells by survivin-directed antisense oligodeoxynucleotides and siRNA. Cancer Lett 232: 243-254, 2006.

32. Fukuda S, Foster RG, Porter SB and Pelus LM: The antiapoptosis protein survivin is associated with cell cycle entry of normal cord blood CD34(+) cells and modulates cell cycle and proliferation of mouse hematopoietic progenitor cells. Blood 100: 2463-2471, 2002.

33. Ryan B, O'Donovan N, Browne B, et al: Expression of survivin and its splice variants survivin-2B and survivin-DeltaEx3 in breast cancer. Br J Cancer 92: 120-124, 2005.

34. Span PN, Tjan-Heijnen VC, Heuvel JJ, De Kok JB, Foekens JA and Sweep FC: Do the survivin (BIRC5) splice variants modulate or add to the prognostic value of total survivin in breast cancer? Clin Chem 52: 1693-1700, 2006.

35. Mahotka C, Krieg T, Krieg A, et al: Distinct in vivo expression patterns of survivin splice variants in renal cell carcinomas. Int J Cancer 100: 30-36, 2002.

36. Fangusaro R, Jiang Y, Holloway MP, et al: Survivin, Survivin-2B, and Survivin-deltaEx 3 expression in medulloblastoma: biologic markers of tumour morphology and clinical outcome. Br J Cancer 31: 359-365, 2005.

37. Taubert H, Kappler M, Bache M, et al: Elevated expression of survivin-splice variants predicts a poor outcome for soft-tissue sarcoma patients. Oncogene 24: 5258-5261, 2005.

38. Belyanskaya LL, Hopkins-Donaldson S, Kurtz S, et al: Cisplatin activates Akt in small cell lung cancer cells and attenuates apoptosis by survivin upregulation. Int J Cancer 117: 755-763, 2005.

39. Williams NS, Gaynor RB, Scoggin S, et al: Identification and validation of genes involved in the pathogenesis of colorectal cancer using cDNA microarrays and RNA interference. Clin Cancer Res 9: 931-946, 2003

40. Geller J, Petak I, Szucs KS, Nagy K, Tillman DM and Houghton JA: Interferon-gamma-induced sensitization of colon carcinomas to ZD9331 targets caspases, downstream of Fas, independent of mitochondrial signaling and the inhibitor of apoptosis survivin. Clin Cancer Res 9: 6504-6515, 2003.

41. Li JX, Zhou KY, Liang T and Zhang YF: Knockdown of survivin expression by small interfering RNA induces apoptosis in human breast carcinoma cell line MCF-7. Ai Zheng 24: 268-272, 2005.

42. Wagner M, Schmelz K, Wuchter C, Ludwig WD, Dorken B and Tamm I: In vivo expression of survivin and its splice variant survivin-2B: impact on clinical outcome in acute myeloid leukaemia. Int J Cancer 119: 1291-1297, 2006.

43. Lai GJ and McCobb DP: Regulation of alternatively splicing of slo $\mathrm{K}^{+}$channels in adrenal and pituitary during the stress hyporesponsive period of rat development. Endocrinology 147: 3961-3967, 2006 